infections in a group of renal transplant recipients. Br f Dermatol 1986;115 $681-92$.

14 de Villiers EM, Gissmann L, zur Hausen H. Molecular cloning of viral DNA from human genital warts. 7 Virol $1981 ; 40: 932-5$

15 Gissman L, Diehl V, Schulz-Coulon HJ, zur Hausen H. Molecular cloning and characterisation of human papillomavirus DNA derived from a laryngeal papilloma. I Virol 1982;44:393-400.

16 Durst M, Gissman L, Ikenberg H, zur Hausen H. A papillomavirus DNA from a cervical carcinoma and its prevalence in cancer biopsy samples from different geographic regions. Proc Natl Acad Sci L'SA 1983;80:3812-5.

17 Boshart M, Gissman L, Ikenberg H, Kleinheinz A, Scheurlen W, zur Hausen H. A new type of papillomavirus DNA, its presence in genital cancer biopsies and in cell lines derived from cervical cancer. EMBO $\mathcal{J}$ 1984;3:

18 Schneider V, Kay S, Lee HM. Immunosuppression as a high-risk factor in the development of condyloma acuminatum and squamous neoplasia of the cervix. Acta Cytol 1983;27:220-4

19 Rotkin ID. A comparison review of key epidemiological studies in cervical cancer related to current searches for transmissible agents. Cancer Research 1973;33:1353-67.

20 Sillman F, Stanek A, Sedlis A, et al. The relationship between human papillomavirus and lower genital intraepithelial neoplasia in immunosuppressed women. Am F Obstet Gynecol 1984;150:300-8.

21 Schneider A, Sawada E, Gissman L, Shah K. Human papillomaviruses in women with a history of abnormal Papaniclaou smears and in their mate partners. Obstet Gynecol 1987;69:554-62.

22 Shield PW, Daunter B, W'right RG. The Pap smear revisited. Aust NZ J Obstet Gunaecol 1987;27:269-82.

(Accepted 31 October 1988)

\title{
Late renal failure due to prostatic outflow obstruction: a preventable disease
}

\author{
S H Sacks, S A J R Aparicio, A Bevan, D O Oliver, E J Will, A M Davison
}

Nuffield Department of Clinical Medicine, John Radcliffe Hospital, Headington, Oxford OX3 9DU

S H Sacks, MRCP, medical tutor

S A J R Aparicio, BA, medical student

Renal Unit, Churchill Hospital, Headington, Oxford OX3 7LJ

A Bevan, $\mathrm{MB}$, senior house officer

D O Oliver, FRCP, consultant physician

Renal Unit, St James's University Hospital, Leeds LS9 7TF

E J Will, MRCP, consultant physician

A M Davison, FRCP, consultant physician

Correspondence to: Dr S H Sacks, Renal Unit, Guy's Hospital, London SEl 9RT.

Br Med f 1989;298:156-9

\begin{abstract}
Nineteen patients presenting with late renal failure due to prostatic outflow obstruction (mean age 68.7 years; mean serum creatinine concentration $1158 \mu \mathrm{mol} / \mathrm{l}$ ) were identified from the admission records of two renal units. As late renal failure secondary to prostatic enlargement is preventable case records were analysed retrospectively in an attempt to identify aspects of management in which preventive efforts might be of value. Delays in referral were common, with a mean of 2.8 years between the onset of prostatic symptoms and time of referral, six patients being referred who had had symptoms for more than three years. Four of five patients who had had a prostatectomy were known to be in renal failure at the time of operation but were not referred until 2-13 years later, when prostatic symptoms had recurred and there was evidence of progressive nephropathy with dilatation of the upper urinary tract. Two patients died on admission and eight ( $47 \%$ of survivors) required long term dialysis, most patients $(80 \%)$ requiring some dialysis support during the initial period.
\end{abstract}

These findings suggest that progressive nephropathy caused by prostatic outflow obstruction might, in part, be averted by more adequate screening of renal function in men with untreated prostatism and closer follow up of patients with uraemia at the time of prostatectomy.

\section{Introduction}

Though prostatic symptoms are known to occur in up to $40 \%$ of men over $65,{ }^{\prime}$ the prevalence of renal failure due to protstatic disease is essentially unknown. Dilatation of the upper urinary tract has been reported in about $5 \%$ of men being investigated in hospital for prostatism,,$^{23}$ and though the pressure-flow relations within the bladder associated with upper tract dilatation have been greatly clarified,,$^{4-10}$ there has been surprisingly little emphasis on the progressive effects on the kidney leading to irreversible renal failure.

Late or end stage renal failure secondary to prostatic outflow obstruction should be amenable to prevention if cases are recognised early and the obstruction relieved. New cases of late or irreversible disease arise each year, however, and figures from the European Dialysis and Transplant Association registry showing that $3-5 \%$ of end stage renal disease in patients over the age of 65 is due to acquired obstruction ${ }^{11}$ indicate that prostatic enlargement may be an important cause of end stage renal disease. The explanation for these late and undiagnosed cases is not clear. It has been suggested that severe obstruction may occur without prostatic symptoms, ${ }^{4}$ but whether lack of symptoms adequately explains these apparent failures in detection or whether there are other aspects of management that might be improved is not clear.

The purpose of this report is to identify aspects of management in which preventive efforts might be of value. By means of retrospective analysis we studied patients with late renal failure referred to two renal units over several years.

\section{Patients and methods}

The study was carried out on patients who were admitted to the renal units at the Churchill Hospital, Oxford, and St James's University Hospital, Leeds. These two units serve populations of about $2 \cdot 4$ million and $2 \cdot 0$ million respectively. Patients with a diagnosis of prostatic hypertrophy and obstructive uropathy or nephropathy were identified from three sources: (a) the databases of the renal units; (b) Hospital Activity Analysis based on diagnosis on admission; and (c) personal recall of cases. These sources were known to be incomplete as not all patients were coded at the time of study and some codings were known to be incorrect. Also patients in Oxford who were managed without dialysis were not entered into the database for chronic renal failure and the aetiological diagnosis was not always made at the time of admission. Patients with congenital obstruction or acquired obstruction not due to prostatic disease were excluded from the study. Cases of obstruction due to infiltration of the lower ureters by prostatic carcinoma were also excluded. The diagnosis of obstruction was confirmed by ultrasonography of the kidneys and urinary tract and in some cases by intravenous urography. Cystoscopy was carried out to confirm the diagnosis and obtain tissue for histological study.

Thirteen patients in Oxford and six in Leeds were identified for further study. Most were admitted between January 1984 and March 1987 (see table). Eleven patients had been referred from regional hospitals outside Oxford, and five patients in Leeds had been referred from other hospitals in the area.

The case notes of patients were examined and information on the following extracted: age at admission; duration of prostatic or uraemic symptoms, or both; interval between seeing a doctor and referral to hospital; treatable conditions on admission (urinary tract infection, hypovolaemia, nephrotoxic drugs, medical conditions contributing to renal failure); whether a diagnosis of prostatic obstruction was made 
by the primary referral centre; size of the prostate; blood biochemical and haemoglobin concentrations; urinary drainage procedure (catheter, nephrostomy); short term mode of dialysis; date(s) of prostatectomy; histological diagnosis; and long term dialysis requirement. All patients except those who died had been followed up for a minimum of six months at the time of study.

Results are expressed as means and (when appropriate) standard deviations. In calculating the mean duration of prostatic or uraemic symptoms the lower figure was used when the duration was expressed as being greater than a certain number of months or years and two years was used when the duration was stated as "a few years."

\section{Results}

The table gives the data on the 19 cases studied. The mean (SD) age of patients at presentation was $68 \cdot 7$ $(7 \cdot 6)$ years (range $52-86$ ) and the mean serum creatinine concentration on admission to hospital 1158 $\mu \mathrm{mol} / \mathrm{l}$. Pelvicaliceal dilatation with dilatation of the ureters to the level of the bladder was confirmed in 17 patients on referral for renal assessment. In one patient (case 15) ultrasound examination was not performed but renal function improved dramatically after relief of obstruction. Another patient (case 5) with bilaterally small kidneys did not show pelvicaliceal dilatation when examined by ultrasound or intravenous urography. Histological studies of prostatic tissue showed that 15 cases were benign and four malignant. In malignant cases there was no invasion of the ureteric orifices or bladder trigone seen at cystoscopy. All cases were acute referrals. The diagnosis of prostatic enlargement causing obstructive nephropathy was not made by the referring team in one third of cases. The prostate was enlarged in all cases.

Presenting symptoms-At the time of admission 16 patients complained of prostatic symptoms (hesitancy, nocturia, poor stream, terminal dribbling). The mean duration of symptoms was 2.8 years (range three months to nine years). Three other patients at first denied difficulty with micturition, but on further questioning it was apparent that prostatic symptoms had been present for some time but had not been regarded as abnormal. The reason for admission in all cases was the recent onset of uraemic symptoms, which had developed over a few days to several months in one case, most patients having had symptoms for "a few weeks."

Previous prostatectomy-Five patients had had a previous prostatectomy, which had been carried out a mean of 5.4 years (range 2-13) before the current admission with renal failure. Four patients were known to have been in renal failure at the time of the first prostatectomy with apparent initial recovery of renal function in three patients for whom data were available. Prostatic symptoms recurred after operation in all cases, however, and we found dilated upper urinary tracts in four patients when they were referred to us. Two other patients were on the waiting list for a prostatectomy at district general hospitals; one of these (case 19) had been waiting for 12 months and the other patient (case 2) had been waiting for an unspecified period at the time of admission.

Reversible factors-Medically treatable conditions which may have contributed to uraemia were noted in eight patients at the time of admission; five were found to have urinary tract infections and three were also noted to be hypovolaemic on arrival. Plasma potassium concentrations $>6 \mathrm{mmol} / \mathrm{l}$ were recorded in eight patients on admission. These may have represented partially corrected values as most patients were received from other hospitals where initial resuscitation had been carried out. The haemoglobin concentration was $>110 \mathrm{~g} / \mathrm{l}$ in three cases, suggesting the possibility of a more acute course of renal failure or of salt or water depletion.

Outcome-There were two immediate deaths (cases 18 and 19) related to uraemia or hyperkalaemia, or both, and two further deaths about six months after admission in patients having long term dialysis. The overall mortality at six months was therefore $21 \%$. Of the late deaths, one was from myocardial infarction (case 10) and the other was from unknown causes (case 4). Of the 17 patients who survived the initial period, eight required maintenance haemodialysis. Nine patients recovered sufficiently after prostatectomy not to need further dialysis, but the mean glomerular filtration rate on "recovery" was only $15 \mathrm{ml} / \mathrm{min}$ (range 6-25) in five patients in whom the creatinine clearance was measured. Altogether 16 patients received some form of dialysis support during management. We analysed outcome ("on" or "off" dialysis six months after admission) in relation to mean age; duration of symptoms; blood urea, creatinine, and haemoglobin concentrations; and occurrence of

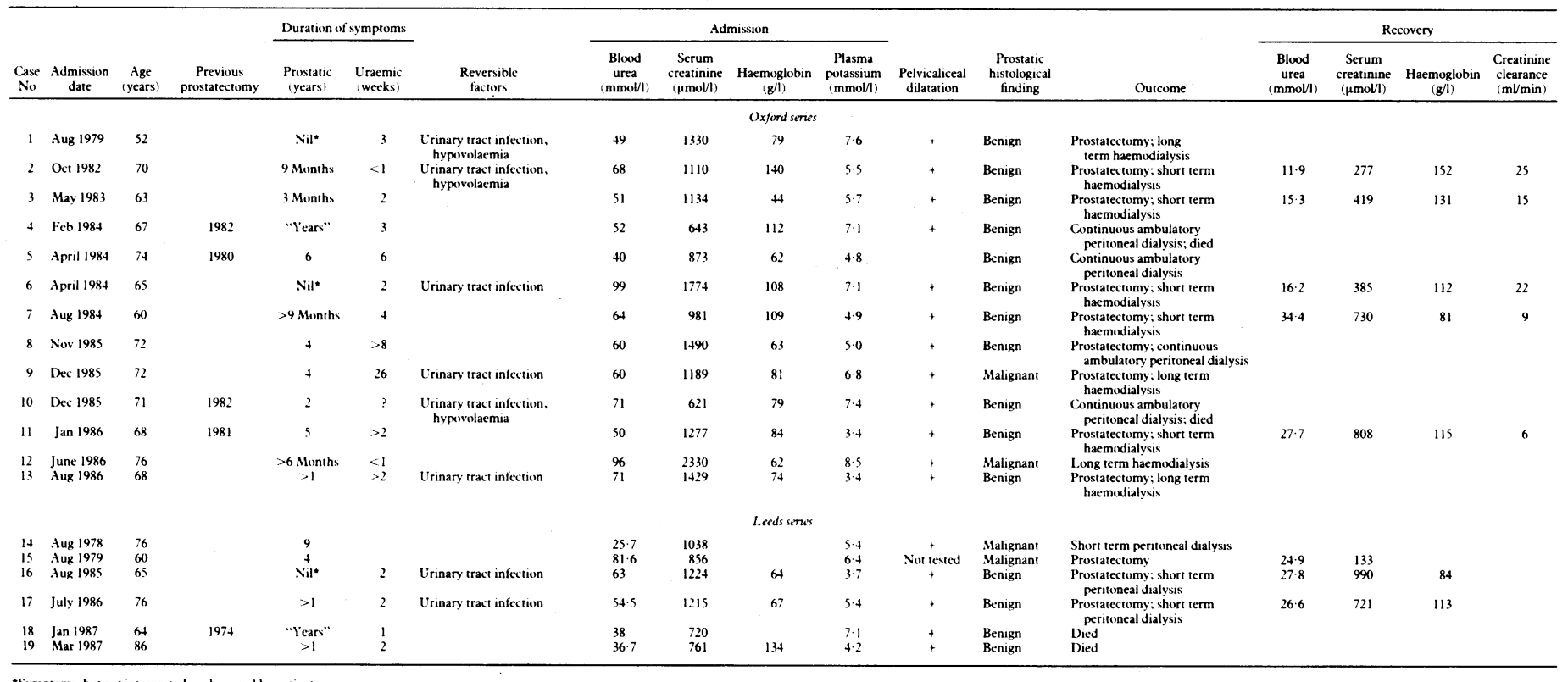


infection or hypovolaemia, or both. Though the small number of cases precluded formal statistical analysis, the eight patients requiring long term dialysis were slightly older (mean age 69 years) than those who regained renal function (mean age 67). There were no substantial differences in the presenting biochemical and haematological values of these two groups, though the haemoglobin concentration at recovery was, as expected, higher in those in whom renal function improved. Initial urinary infection and hypovolaemia occurred with similar frequency in both groups. There appeared to be no correlation between the duration of prostatic symptoms and renal outcome.

\section{Discussion}

This study examined the possibility of preventing late renal failure due to prostatic outflow obstruction by analysis of 19 cases (15 benign, four malignant) identified from the admission records of two renal units. All patients had prostatic symptoms, and five had had a previous prostatectomy with early recurrence of symptoms and evidence of progressive nephropathy after operation. Hydroureteronephrosis was found in 17 cases, and in one case in which imaging of the urinary tract was not carried out prompt recovery of renal function occurred after relief of obstruction. Patients with obstruction due to infiltration of the lower ureters or bladder trigone by prostatic carcinoma were excluded from the study. The present cases were therefore consistent with obstruction at the level of the bladder neck resulting in dilatation of the bladder and upper urinary tract, with compression and thinning of the renal cortex and eventual renal failure.

Twelve of the 19 patients had had prostatic symptoms for more than 12 months by the time of referral to hospital, and six had had symptoms for more than three years. As the severity of renal impairment and the degree of recovery of function after relief of obstruction are influenced by the duration (and completeness) of obstruction, not surprisingly renal failure was to a great extent irreversible by the time of admission to hospital; eight patients required long term dialysis and only marginal recovery of function occurred in others, some of whom were also at risk of becoming dependent on dialysis. In addition there were two immediate deaths from the effects of uraemia. Arguably, therefore, one of the most important factors to affect morbidity was delayed diagnosis.

An important difficulty is in recognising which men with prostatism are at risk of upper tract dilatation and therefore require further investigation. A history of enuresis, painless chronic retention, and a palpable bladder should suggest a diagnosis of high pressure chronic retention with its attendant risk of hydroureteronephrosis. $^{78}$ Several studies, however, have shown that prostatic symptoms bear little relation to the degree of obstruction. Whether the patient has classic "obstructive" symptoms (hesitancy, weak stream) or classic "irritative" symptoms (frequency, nocturia) or whether symptoms are graded according to severity appears not to predict the degree of obstruction to urinary flow. ${ }^{8-10} 12$ Similarly, assessment of prostatic size, though confirming a diagnosis of prostatism, is notoriously unreliable in assessing obstruction..$^{90^{12} 13}$ It is always necessary to look for symptoms and signs of chronic renal failurefor example, nausea, lethargy, oedema, and hypertension $^{1+}$ - but these occur at a late stage, usually when irreversible damage has been done.

Obstruction may be confirmed by imaging of the renal tract (by ultrasonography or intravenous urography), assessing renal function, ${ }^{13}$ or finding high bladder pressures on urodynamic investigation. ${ }^{6}$ Much discussion has centred on the role of these as routine investigations. de Lacey et al have suggested that imaging of the renal tract should be undertaken only if it is decided that prostatectomy is not warranted by the symptoms. ${ }^{3}$ They argue that the management of patients who have already been accepted for surgery is unlikely to be influenced by showing dilated upper tracts preoperatively. Others have concluded that visualisation of the urinary tract remains a sound principle of management. ${ }^{15}$ Our study suggests that even patients who have prostatectomy are at risk of subsequent renal failure, and it seems important that those with upper tract dilatation should be identified at the time of surgery and followed up carefully.

A more difficult question arising from this study is whether some form of screening is necessary in primary health care. If cases such as the ones that we saw are not being identified should, for example, all patients who consult their general practitioners with prostatic symptoms have renal ultrasonography or should they have blood sent for urea and creatinine measurements? The argument depends on how frequently renal impairment might be expected to occur as a complication of prostatic disease and also on the cost effectiveness of detection and treatment. We emphasise that though renal failure of the severity documented here is rare, lesser degrees of functional impairment with hydronephrosis occur and the prevalence of these is essentially unknown. Various studies have suggested that renal impairment with hydronephrosis is present in about $5 \%$ of cases under investigation, ${ }^{23}$ but no comparable figures areavailable in primary health care. In an analysis of 379 patients having prostatectomy in Oxford we found $27(7 \%)$ who had a plasma creatinine concentration greater than $200 \mu \mathrm{mol} / \mathrm{l}$ at the time of surgery (unpublished). These observations indicate that obstructive nephropathy secondary to prostatic disease is perhaps more common than is widely perceived.

It would clearly be unfeasible to perform ultrasonography on all men consulting a doctor for prostatic symptoms or to refer all cases to hospital. Measurement of blood urea and creatinine concentrations, on the other hand, is widely available and would allow detection of cases of moderate to severe (but not mild) renal impairment. We are at present evaluating a programme of symptomatic and biochemical screening of 3000 men in the Oxfordshire community with the aim of identifying those with renal failure due to prostatic obstruction. Even if the number of cases identified is small it is a preventable disease, and, moreover, at a current cost of around $£ 14000$ to maintain one patient with hospital haemodialysis for one year ${ }^{16}$ the saving would be considerable.

Renal failure after prostatectomy may be the result of incomplete removal of obstruction, stone formation or infection in persistently dilated upper tracts, or progressive loss of nephrons due to hyperfiltration of remnant nephrons. ${ }^{17}$ Stones were not found in any of our cases. Eight of our 19 patients had a urinary infection at the time of admission, but the role of secondary infection in the pathogenesis of chronic renal failure remains unclear. The weight of the evidence presented here, with early recurrence of prostatic symptoms, progressive nephropathy, and presence of dilated upper urinary tracts in four of five patients, is more consistent with continued or recurrent obstruction. It is important that these cases are recognised at the time of prostatectomy and scrupulously followed up.

It has been stated that men with mild or moderate prostatic symptoms may be left untreated for several years without any harmful effects. ${ }^{18} 19$ Though this may be so in most cases, this study emphasises the severe complications of late or undiagnosed urinary tract 
obstruction due to prostatic disease. A prime consideration in those who are not referred for surgery should be identification of cases at risk by careful abdominal palpation and inquiry about enuresis - and perhaps by more thorough screening of renal function in patients with otherwise minor symptoms.

We are grateful to Ms Penny Wright and Ms Sarah-Jane Metson for preparing the manuscript.

1 Gotin R. The health status of elderly men: a community study. Public Health 1982;96:345-54.

Abrams PH, Roylance J, Feneley RCL. Excretion urography in the investigation of prostatism. $\mathrm{Br} f$ Urol 1976;48:681-4.

3 de Lacey G, Johnson S, Mee D. Prostatism: How useful is routine imaging of the urinary tract? Br Med f 1988;296:965-7.

4 George NJR, Feneley RCL, Roberts JBM. Identification of the poor risk patient with "prostatism" and detrusor failure. Br $\mathcal{F}$ Urol 1986;58:290-5.

5 George NJR, O'Reilly PH, Barnard RJ, Blacklock NJ. High pressure chronic retention. Br Med F 1983;286:1780-3.

6 George NJR, O'Reilly PH, Barnard RJ, Blacklock NJ. Practical management of patients with dilated upper tracts and chronic retention of urine. Brf Urol 1984;56:9-12.

7 O'Reilly PH, Brooman PJC, Farah NB, Mason GC. High pressure chronic retention. Incidence, aetiology and sinister implications. Br $\mathcal{A}$ Urol 1986;58: $6+4-6$.
8 Styles RA, Ramsden PD, Neal DE. Chronic retention of urine. The relationship between upper tract dilatation and bladder pressure. $\mathrm{Br} f \mathrm{Urol}$ 1986;58:647-51.

9 Jenson KME, Jorgensen BJ, Mogensen P, Bille-Brahe NE. Some clinical aspects of uroflowmetry in elderly males. Scand $\mathcal{F}$ Urol Nephrol 1986;20: 93-9.

10 Warwick RT, Whiteside CG, Arnold EP, et al. A urodynamic view of prostatic obstruction and the results of prostatectomy. $\mathrm{Br} \mathcal{F}$ Urol 1973;45:631-45.

11 European Dialysis and Transplant Association. Combined report on dialysis and transplantation, XV. Proceedings of the European Dialysis and Transplant and transplantation, XV. Prociation 1985;22:3-54.

12 Birkhoff JD. Natural history of benign prostatic hypertrophy. In: Hinman F Jr, ed. Benign prostatic hypertrophy. New York: Springer Verlag, 1983:1-9. 13 O'Reilly PH. Assessment for prostatectomy. Br.Med f 1987;294:1370-1.

14 Jones DA, George NJR, O'Reilly PH, Barnard RJ. Reversible hypertension associated with undiagnosed high pressure chronic retention. Lancel 1987;i:1052-4.

15 Pinck BD, Corrigan MJ, Jasper P. Pre-prostatectomy excretory urography Does it merit the expense? $\mathcal{F}$ Urol 1980;123:390-3.

16 Wood IT, Mallick NP, Wing AJ. Prediction of resources needed to achieve the national target for treatment of renal failure. Br Med f 1987;294:1467-70.

17 Dunn BR, Anderson S, Brenner BM. The haemodynamic basis of progressive renal disease. Seminars in Nephrology 1986;6:122-33

18 Craigen AA, Hickling JB, Saunders CRG, Carpenter RG. Natural history of prostatic obstruction. I $R$ Coll Gen Pract 1969;18:226-32.

19 Ball AJ, Fenely RCL, Abrams PH. The natural history of untreated "prostatism." Br f Urol 1981;53:613-6.

(Accepted 2 November 1988

\title{
What do psychiatric inpatients really want?
}

\author{
Keith McIntyre, Michael Farrell, \\ Anthony S David
}

University of Glasgow, Glasgow G12 8QQ

Keith McIntyre, medical student

The Bethlem Royal and Maudsley Hospitals, London SE5 8AZ

Michael Farrell, MRCPSYCH, senior registrar

\section{Institute of Psychiatry,}

London SE5 8AF

Anthony S David,

MRCPSYCH, research worker

Correspondence to:

Dr David.

BrMed f 1989:298:159-60
In the age of the consumer the customer's view is all important. Psychiatric patients are perhaps the last people to be consulted about these matters. This is reflected in the dearth of research on the topic in Britain, though it has drawn attention in the United States. ' Patients' attitudes to specific aspects of their treatment ${ }^{23}$ have been recorded, as have views on the hospital milieu.+ The purpose of this study was to obtain the views of current inpatients at an inner London psychiatric teaching hospital on how helpful they found the various components of hospital care.

\section{Patients, methods, and results}

All patients from seven wards who had been in hospital for more than one week were approached. From a total sample of 117 , seven were too disturbed to be interviewed and 11 refused, leaving 99 patients (54 women, 45 men) who agreed to participate. Twenty eight patients were in a psychiatric hospital for the first time and 24 were being detained compulsorily under the Mental Health Act. Diagnoses according to the International Classification of Diseases (9th revision) were: schizophrenic psychoses 42 ; mania 13 ; neurotic depression 18; alcohol or drug dependence, or both, 8 ; personality disorder 7 ; organic disorders 8 ; and three patients with other neurotic disorders. Ninety three patients were receiving drug treatment, 46 taking neuroleptics.

All patients were given a simple structured interview lasting 15 minutes. The interviewer was not introduced with a title-for example, doctor or nursethus avoiding biasing the responses. Patients were encouraged to answer freely and in confidence. The interview contained 10 questions requiring a judgment of "helpfulness" on a five point scale (0-4) ranging from "not at all helpful" to "a little," "quite," "very," and "extremely helpful." Items of "helpfulness" were (1) talking to the doctor, (2) talking to the nurse, (3) talking to other patients, (4) ward group (regular meetings composed of patients and staff), (5) occupa- tional therapy, (6) drug treatment, (7) the ward round, (8) just being in hospital, (9) visitors, and (10) free pass (permission to leave the ward). The patients' estimate of the time engaged in these activities was noted. Each subject was rated by his or her ward nurse or doctor on the global assessment scale, ${ }^{5}$ a measure of overall psychiatric disturbance scored from 0 (completely disabled) to 100 (normal).

The mean age of the sample was $36 \cdot 1$ (SD 13.7) years. Mean length of stay was $21 \cdot 0(\mathrm{SD} 31 \cdot 1)$ weeks, mode three weeks. Thus the distribution was predictably skewed, most patients staying a brief period in hospital but substantial numbers staying much longer. Average score on the global assessment scale was $59 \cdot 2$ (SD $17 \cdot 0$ ). The table shows the mean scores for each item of helpfulness and the rank order of items.

Patients' attitudes to hospitalisation and treatment in order of perceived helpfulness $(n=99)$

\begin{tabular}{lcc}
\hline \multicolumn{1}{c}{ Item } & Mean score $(\mathrm{SD})$ & No studied \\
\hline (1) Free pass & $2 \cdot 79(1 \cdot 03)$ & 77 \\
(2) Visitors & $2 \cdot 47(1 \cdot 13)$ & 88 \\
(3) Talking to doctor & $2 \cdot 34(1 \cdot 17)$ & 99 \\
(4) Talking to nurse & $2 \cdot 33(1 \cdot 17)$ & 99 \\
(5) Drug treatment & $2 \cdot 19(1 \cdot 32)$ & 93 \\
(6) Hospital & $2 \cdot 02(1 \cdot 36)$ & 99 \\
(7) Occupational therapy & $2 \cdot 01(1 \cdot 31)$ & 85 \\
(8) Ward round & $1 \cdot 91(1 \cdot 27)$ & 80 \\
(9) Other patients & $1 \cdot 83(1 \cdot 13)$ & 99 \\
(10) Group & $1 \cdot 08(1 \cdot 09)$ & 93 \\
\hline
\end{tabular}

When involuntary patients were excluded from the analyses the only substantial change in the judgments about hospitalisation was that visitors assumed less importance. Those who were rated less ill on the global assessment scale found talking to the doctor and going to occupational therapy more helpful (Pearson's correlation: $r=0.24 ; p<0.01$ ). Increasing age had the same effect. Severity, however, did not influence the perception of the nurses' helpfulness $(r=0.02 ; \mathrm{NS})$ or any other variables. There was a non-significant trend for women to find talking to the nurse more helpful than talking to the doctor (unpaired $t$ test, $\mathrm{p}=0 \cdot 1$ ). Also women found visitors and a free pass less helpful $(p<0.05)$ than did men. Surprisingly, patients admitted for the first time had similar opinions to those who had been admitted before. Similarly, length of stay did not correlate with attitudes to treatment but bore an inverse relation to how helpful other patients were perceived to be $(r=-0 \cdot 2 ; p=0 \cdot 02)$. Comparing 